\title{
Téoros
}

Revue de recherche en tourisme

\section{Femmes, coeur de cible et construction d'un discours promotionnel}

\author{
Le cas du tourisme de bien-être
}

\section{Marie-Ange Bugnot, Carmen Cortés et Isabel Turci}

Volume 29, numéro 2, 2010

Tourisme et femmes

URI : https://id.erudit.org/iderudit/1024876ar

DOI : https://doi.org/10.7202/1024876ar

Aller au sommaire du numéro

Éditeur(s)

Université du Québec à Montréal

ISSN

0712-8657 (imprimé)

1923-2705 (numérique)

Découvrir la revue

Citer cet article

Bugnot, M.-A., Cortés, C. \& Turci, I. (2010). Femmes, coeur de cible et construction d'un discours promotionnel : le cas du tourisme de bien-être. Téoros, 29(2), 103-111. https://doi.org/10.7202/1024876ar
Résumé de l'article

Les magazines de tourisme et de voyage utilisent un discours verbal apparemment neutre. Pourtant, une analyse qualitative du discours promotionnel, véhiculé aussi bien par le texte journalistique que par la publicité payante, nous permet de révéler la nature des mécanismes d'accroche et de persuasion, c'est-à-dire les composantes iconiques et textuelles qui interpellent très particulièrement les femmes. En effet, les discours des magazines vont montrer de subtiles marques de genre à travers lesquelles le public féminin peut se reconnaître. Nous avons circonscrit notre corpus aux textes consacrés aux prestations de bien-être, où nous avons dégagé des modèles de comportement et des tendances nouvelles ou revisitées qui nourrissent l'architecture du sens. La quête du bonheur guide l'expérience individuelle dans un parcours dépaysant qui conjugue renouveau, jouvence et beauté. Des réseaux thématiques, dont nous étudions les plus représentatifs, s'entrelacent pour orienter l'interprétation du message en vue de persuader le coeur de cible.
Ce document est protégé par la loi sur le droit d'auteur. L'utilisation des services d’Érudit (y compris la reproduction) est assujettie à sa politique d'utilisation que vous pouvez consulter en ligne.

https://apropos.erudit.org/fr/usagers/politique-dutilisation/ 


\title{
Femmes, cœur de cible et construction d'un discours promotionnel Le cas du tourisme de bien-être
}

\author{
Marie-Ange BUGNOT \\ Professeure, Université de Malaga (Espagne), \\ Facultad de Filosofía y Letras \\ mabugnot@uma.es \\ Carmen CORTÉS \\ Professeure, Université de Malaga (Espagne), \\ Facultad de Filosofía y Letras \\ ccortes@uma.es
}

\author{
Isabel TURCI \\ Professeure, Université de Malaga (Espagne), \\ Facultad de Filosofía y Letras \\ miturci@uma.es
}

\begin{abstract}
RÉSUMÉ: Les magazines de tourisme et de voyage utilisent un discours verbal apparemment neutre. Pourtant, une analyse qualitative du discours promotionnel, véhiculé aussi bien par le texte journalistique que par la publicité payante, nous permet de révéler la nature des mécanismes d'accroche et de persuasion, c'est-à-dire les composantes iconiques et textuelles qui interpellent très particulièrement les femmes. En effet, les discours des magazines vont montrer de subtiles marques de genre à travers lesquelles le public féminin peut se reconnaître. Nous avons circonscrit notre corpus aux textes consacrés aux prestations de bien-être, où nous avons dégagé des modèles de comportement et des tendances nouvelles ou revisitées qui nourrissent l'architecture du sens. La quête du bonheur guide l'expérience individuelle dans un parcours dépaysant qui conjugue renouveau, jouvence et beauté. Des réseaux thématiques, dont nous étudions les plus représentatifs, s'entrelacent pour orienter l'interprétation du message en vue de persuader le cœur de cible.
\end{abstract}

Mots-clés: Femme, publicité, promotion, discours.

Les magazines classés "tourisme et voyage», comme bien d'autres types de publications de la presse magazine à caractère promotionnel, s'adressent spécialement aux femmes. Cependant, une différence saillante sépare ceux-ci de la plupart de leurs concurrents sur le marché : à première vue très peu d'indices verbaux nous avertissent qu'ils s'adressent à ce cœur de cible. Nous nous sommes donc demandé par quels moyens ils arrivent à interpeller les femmes et à les retenir, quelles sont les stratégies et les structures rhétoriques mises en place pour les persuader à agir, pour les pousser à la consommation aussi bien de la publication elle-même que des services et produits qu'on y propose.

Un peu partout dans le monde, des études ont été menées sur le rôle des femmes dans la publicité, sur la construction de leur image dans les médias, sur les stratégies rhétoriques mises en œuvre pour la véhiculer (Perret, 2003; Wykes et Gunter, 2005; Ribas et Todolí, 2008; Reiser et Gresy, 2008; Cortese, 2008; Bugnot, 2009). Or, jusqu'ici aucune étude préalable n'a abordé le sujet de recherche qui nous occupe pour deux raisons. D'une part, pour ce qui est des magazines, les corpus étudiés appartiennent à ceux classés féminins grand public, féminins haut de gamme, people, ou santé et famille. De l'autre, la plupart de ces analyses ne s'occupent que de la publicité payante, dont le discours (texte et icônes) y est d'habitude marqué du point de vue du genre.

Dans les premières années de la décennie 2000, quelques travaux sur les contenus promotionnels dans les magazines féminins ont souligné l'énorme poids du rédactionnel qui complète, nuance et même relaie la publicité payante dans ces médias (Maurin, 2003; Caliandro, 2004). Les magazines deviennent ainsi des objets promotionnels dans leur ensemble. Ce sont les implications de cette idée sur les agencements promotionnels, sur les adresses "genrées", sur les composantes de l'énonciation et sur la construction du sens qui ont guidé plusieurs de nos travaux et qui sous-tendent cette recherche.

Nous avons restreint notre corpus à un type de services, les services de bien-être, dont l'offre et la demande ont fortement augmenté pendant la dernière décennie (Global Spa Summit, 2010). Des causes multiples — sociales, démographiques, économiques et culturelles - expliquent l'énorme dynamisme de ce secteur dans le monde (Ross, 2001; Fontanari et Kern, 2003; García-Altés, 2005). Nous avons spécialement retenu certains phénomènes sociaux qui nous semblent intéressants puisqu'ils sont à la base de nombre de structures 
promotionnelles présentes dans notre corpus : les contraintes de la vie contemporaine dont dérivent de plus en plus de troubles physiologiques et psychiques; le désir de prolonger à la maison les bienfaits obtenus pendant le voyage (Smith et Puczkó, 2009 : 142); le retour en force de l'hédonisme, mode de vie individuel et forme de représentation collective; le refus de vieillir qui s'accorde au souhait de continuer à séduire; et finalement, la tendance des consommateurs à amalgamer les produits provenant de secteurs auparavant séparés, tels la beauté, l'hygiène, la santé ou l'alimentation (Mermet, 2010).

\section{Femmes, tourisme et bien-être}

Les femmes appartenant aux classes aisées jouissaient autrefois des bienfaits de la saison qui, à partir du Second Empire, représenta un haut fait social réunissant les sphères gouvernementales, les acteurs économiques et autres cercles d'influence français. Les femmes y voyaient un lieu de mixité sociale où les contraintes qui leur interdisaient la liberté de leur corps ou le choix de certaines activités de loisir se libéraient. Dans ce sens, Wallon (1981 : 226) confirme que "[1]es dames doivent, pendant la durée de leur cure, renoncer à l'usage du corset»; affiches et lithographies font d'elles l'image des marques de bicyclette et d'automobile; elles s'adonnent à des sports traditionnellement masculins, tel le tir aux pigeons (Gros, 1899, cité dans Dutheil, 2003 : 42). De nos jours, les femmes deviennent de plus en plus des usagers des services touristiques et sont «les principaux décideurs, les premiers prescripteurs» des dépenses de la famille (Rougès, 2009; Euromonitor international, 2009a et 2009b), notamment des dépenses de voyage (L’Assemblée des femmes, 2003).

L'expression «tourisme de bien-être» est en quelque sorte redondante puisque tout déplacement touristique est censé apporter par lui-même des bienfaits personnels et sociaux qu'on identifie comme du bien-être (Proulx, 2005). Or, le choix des services bien-être en est une recherche consciencieuse, voire délibérée. Le bien-être est un phénomène complexe (Smith et Puczkó, 2009 : 54-58), un processus de prise en charge de soi qui comprend le bien-être physique, émotionnel, mental et spirituel, où le social et la nature jouent un rôle important.

L'industrie touristique spécialisée dans ce secteur essaie de répondre aux attentes du public en développant des produits tels le spa, les massages, les traitements corporels ou les diètes (Chen et al., 2008 : 106-108). Les clients sont très souvent des femmes à hauts revenus mais à court de temps (Smith et Puczkó, 2009 : 77-78), pour qui la relaxation du corps et de l'esprit, la pratique d'activités diverses, les loisirs et le contact avec la nature constituent les motivations principales (Chen et al., $2008: 111$ ).

Le discours promotionnel du tourisme bien-être, ciblant depuis des années les femmes, a récemment élargi son champ d'action aux hommes, notamment au public homosexuel, frange d'un marché au pouvoir d'achat élevé (Tissier-Desbordes et Ulrich, 2006), une évolution qui rappelle facilement la trajectoire connue par les produits de beauté. Pour ce qui est du marché du bien-être en France, $59 \%$ des femmes contre $37 \%$ des hommes ont plébiscité en 2009 pour des établissements tels que les spas, les centres de thalassothérapie, les hammams, etc., comme lieux de détente (IPSOS, 2009). Ceci dit, la fréquentation des établissements de bien-être est prioritairement féminine - $70 \%$ pour les centres de thalassothérapie en France, 49 \% des femmes entre 25 et 49 ans habitant en milieu urbain pour ce qui est des spas (Thermalies, 2010). Le marché féminin du produit bien-être semble bien plus consolidé que celui des hommes, qui ont incorporé cette pratique à leurs loisirs plus lentement étant donné qu'ils " are much more oriented towards the activity and the visit » (Wearing et Wearing, 1996; cité dans Pritchard et Morgan, $2000: 899$ ).

\section{Corpus}

Notre corpus est composé du magazine bimestriel L'Art de Voyager publié par Lefebvre (42 numéros apparus entre les années 2000 et 2006 dont 17 contenant des textes et/ou des publicités sur le tourisme de bien-être) et du mensuel Bon Voyage publié par Hachette (36 numéros correspondant aux années 2002-2004 dont 13 contenant des textes et/ou des publicités sur le tourisme de bien-être). Dans ce cas-ci, la restriction temporelle du corpus est causée par l'impossibilité d'accéder d'une part aux numéros correspondant aux années 2000-2001 et d'autre part à la disparition de la publication. L'ensemble représente un total de 81 discours journalistiques (notes, articles et dossiers) et 47 publicités et publireportages payants qui se rapportent au tourisme de bien-être. Ces deux titres cultivent un certain art de vivre et proposent des voyages et des séjours haut de gamme. Dans notre texte, les références à ces sources primaires sont présentées selon le format suivant : l'abréviation « $\mathrm{AV} »$ pour L'art de voyager, «BV» pour Bon voyage.

Smith et Puczkó (2009 : 7) ont divisé le domaine du tourisme de santé en deux grands sous-ensembles : le tourisme médical qui exige des services médicaux « durs» et le tourisme de bien-être. Celui-ci comprend l'holisme (pratiques spirituelles, yoga et méditation, et new age), les loisirs (traitements de beauté, sports, entraînement physique et détente) ainsi qu'une zone frontalière avec le médical, le bien-être médical (loisirs thérapeutiques, rééducation, bien-être actif, thalassothérapie, et des programmes nutritionnels et de détoxication). Or, les références aux maladies se trouvent bannies de nos textes, sauf pour ce qui est du stress et des conséquences de la vie active; les cures ont pour objet la remise en forme, le rajeunissement, la compensation des agressions de la vie contemporaine et la beauté. C'est un monde idyllique, parfait, paradisiaque, idéal, qui campe dans les textes et les images, et non seulement dans ceux que l'on considère strictement publicitaires, les espaces payants, puisque c'est surtout le discours rédactionnel (textes et images) qui exhibe des produits qui incitent à l'action, à la consommation, par le biais de la persuasion.

\section{Construction et interprétation du sens dans les magazines "tourisme et voyage»}

Pour essayer de répondre à la question que nous nous étions posée, il nous fallait préalablement appréhender le fonctionnement sémiotique des produits analysés. Or, la construction et la compréhension du sens sont des opérations complexes, déterminées d'un côté par le support (livre, site web, 
journal, etc.) et le public cible, de l'autre côté par le sujet récepteur, l'interprète du message. Deux perspectives sémiotiques complémentaires, celle d'Umberto Eco et celle de Charles Sanders Peirce, permettent de dévoiler le processus de constitution du produit magazine et le mode d'interaction avec l'interprète des discours promotionnels.

Pour ce qui est de leur construction, chacun des magazines fonctionne comme un code tel qu'il est compris par Umberto Eco (1991 : 84-85) : un système qui propose des règles pour générer des signes. Dans chaque numéro, ce code donne lieu, dans un processus dynamique, à des unités formelles et de contenu (article, page, section, etc.) de plus en plus complexes à partir des unités minimales (mots, images, typographie, blancs, etc.). Ces règles de génération sont fixées d'après des critères éditoriaux et adaptées en dernière instance aux attentes du public cible, lui-même sous l'emprise de l'habitus, l'ensemble des goûts, des comportements et des tendances qui sont propres à un groupe et qui rendent cohérentes et légitiment les actions individuelles ou collectives (Bourdieu, 1990 : 88-89). Le code détermine donc la construction intrasémiotique du texte et des images en tant que systèmes de signes considérés séparément. On peut observer, par exemple, comment un réseau de substantifs et de verbes appartenant à des champs sémantiques qu'on peut facilement rapprocher (le plaisir, l'évasion, le rêve) crée progressivement dans le texte une ambiance propice à la détente, à l'abandon, annoncée par le titre, «Le Gellert [sic], source de volupté », dans l'article consacré à cet hôtel situé à Budapest : "volupté des vapeurs», "[s]'abandonner à des moiteurs tropicales ", "nouvelle forme de ravissement», "le lieu tient du décor hollywoodien. Un pur moment de cinéma », «... tout ici invite à l'oubli total. Qu'importe le reste du monde!», "Versant solaire de la rêverie au Gellért» (AV, 1999-2000 : 86). Pour ce qui est des images, nous faisons référence aux rapports formels et de contenu qu'elles établissent entre elles et qui donneraient lieu à des interprétations orientées. Prenons l'exemple d'une page où nous trouvons à droite l'image d'un bassin d'eau cristalline entouré d'une baie vitrée baignée par le soleil, à gauche l'image d'un sauna lumineux et accueillant, et finalement tout en bas à droite celle d'une jeune femme allongée, entourée d'une serviette, les yeux fermés, un petit sourire au coin des lèvres. Le code donne aussi lieu aux relations intersémiotiques présentes sur la page entre ces deux ensembles de signes.

De son côté, la conception peircienne des signes éclaire l'utilisation promotionnelle des magazines. Le signe est, pour Peirce, un amalgame de trois composantes, dont une, l'interprétant, est le produit des rapports avec d'autres signes qui complètent ou déterminent sa signification. Généralement, ces rapports sont choisis par l'interprète en fonction de ses habitudes interprétatives dans un contexte donné (EveraertDesmedt, 2006). Les deux autres termes sont : le «representamen », qui représente l'objet mais qui est pure potentialité, qu'il faut interpréter, par exemple un mot tel qu'il est écrit ou prononcé; l'objet, ce que le signe représente. Pour interpréter le signe, l'interprète considère le "representamen ", qui déclenche un interprétant, c'est-à-dire des associations qui lui permettent l'interprétation.
Un grand nombre de ces rapports se trouvent sur les pages des magazines eux-mêmes, dans le contexte textuel et iconique. Le discours persuasif canalise et multiplie ces connexions interprétatives grâce à l'évocation, à la suggestion, aux valeurs connotatives soulevées par les textes et les images. Il déclenche de ce fait les désirs des consommatrices et des consommateurs, qui ne sont plus seulement des interprètes, mais «des acteurs en situation» mus par des produits qui sont ainsi eux-mêmes devenus des actants, des faits sociaux (Le Boeuf, 2000 : 50-52).

\section{Approches méthodologiques : l'analyse du discours et la sémiotique visuelle}

Par contre, ni les signes pris de façon unitaire ni la compréhension du mécanisme général de création des systèmes de signes à partir d'un code ne pouvaient nous donner la clef des mécanismes persuasifs, car leur fonction pragmatique est indissociable de la sémantique et de la syntaxe (EveraertDesmedt, 1990 et 2006). Nous devions donc considérer et étudier les paradigmes sémantiques et les relations syntaxiques qui forment l'agencement argumentatif des magazines, c'est-à-dire l'action de guidage des interprètes vers des interprétants relativement stables et pertinents, découlant des règles de génération. C'est grâce à une analyse qualitative aussi bien du discours que des images que nous y sommes parvenues.

Pour ce qui est du verbal, nous avons d'abord déterminé, l'analyse morphologique aidant, le ou les sujets de l'énonciation pour chaque article et chaque publicité, ce qui n'a pas toujours été possible puisque les articles sont rarement signés, ainsi que le caractère marqué ou non marqué du point de vue du genre du discours verbal. Nous avons examiné ensuite l'enchaînement syntaxique des unités, ainsi que leurs caractéristiques et groupements sémantiques. C'est ainsi que nous avons constaté l'apparition réitérée de mots, de dénominations, et de réseaux sémantiques qui montrait la récurrence de quatre argumentaires (l'exotisme, la sacralisation de l'espace bien-être, l'emprise de la tradition, la femme nouvelle) qui seraient censés être efficaces du point de vue de la persuasion du public cœur de cible et dont nous analysons les principales composantes.

Pour l'étude des images, nous avons adopté la sémiotique standard de l'École de Paris qui cherche à savoir «ce qui se passe «sous les signes» ou «entre les signes», ce qui est à la base de leurs mutuelles relations d'où jaillit le sens avec toutes les nuances»(Courtés, s. d.). Dans le cas des signes iconiques, cette sémiotique structurelle fondée sur les travaux de Greimas considère trois niveaux d'analyse pour ce qui est du contenu : figuratif (analogies et opposition entre les figures, leur organisation en motifs), narratif (conjonction et disjonction des sujets et des objets, actions et transformations) et thématique (valeurs véhiculées). La compréhension de l'expression plastique passe par l'étude des contrastes dans les formes et par l'analyse des phénomènes concernant la lumière et l'espace dans les compositions (Everaert-Desmedt, 2000). 


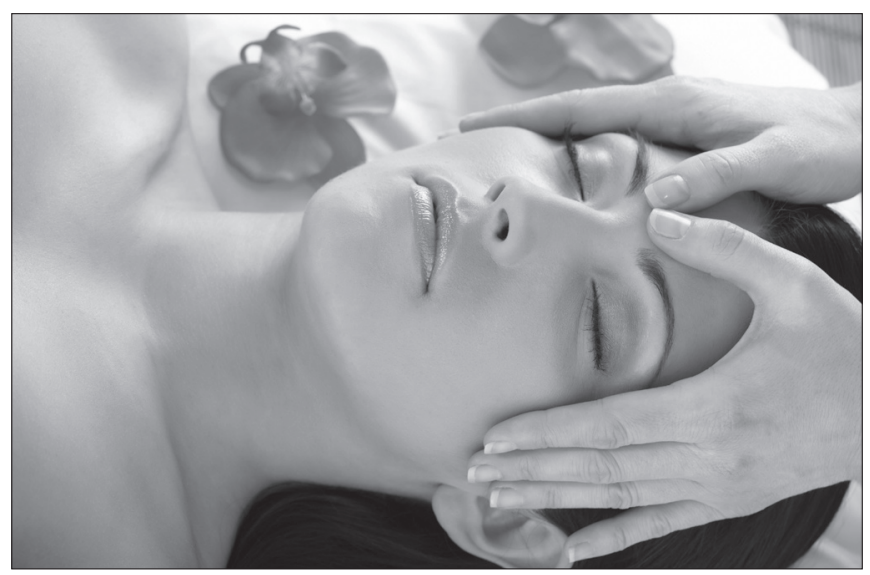

ILLUSTRATION 1 : Les soins et la détente

(photo : Royalty Free Stock Photo, Amaviael).

\section{Discours marqués et discours non marqués : \\ les textes}

Le discours promotionnel qui vante les bienfaits des spas pour hommes, même s'il véhicule l'image d'un nouvel homme soucieux de son apparence et de sa santé, contrebalance très souvent le frein de certains lié à une prétendue féminisation qui pourrait nuire à la consommation par la mise en valeur de la virilité exacerbée du mâle, du macho. La cible est segmentée par le genre sans ambiguité, une stratégie qui a donné ses fruits puisque «l'industrie du spa doit désormais $25 \%$ de son chiffre d'affaires aux consommateurs masculins» (Chevrier, 2006).

Dans les magazines de tourisme et de voyages étudiés, nous avons en général affaire à un discours neutre qui n'intègre que de façon exceptionnelle la marque du féminin, ce qui incite à croire au premier abord que la cible que l'on prétend atteindre n'est pas segmentée par le genre.

Nous trouvons tout de même quelques exemples de textes marqués. Des journalistes ou des écrivaines apportent le témoignage de leur expérience suite à un séjour qui leur a permis de tester pour les magazines certains établissements de bien-être. D'autres écrits-témoignages sont anonymes, mais l'accord au féminin des adjectifs ou des participes passés permet d'attribuer un référent féminin au «Je» communiquant. Ces voix féminines autorisées de par leur connaissance des prestations auxquelles elles se sont initiées assument le rôle discursif de garant de la vérité des dires, une ressource courante du discours persuasif.

Le discours est aussi marqué lorsqu'il fait référence à des soins exclusifs pour les femmes comme la remise en forme postgrossesse ou aux troubles inhérents à la physiologie féminine, tels que l'ostéoporose, plus fréquente chez les femmes $\left(\mathrm{BV}, 2003, \mathrm{n}^{\circ} 28: 52\right)$, les soins anticellulite (BV, 2003, $\mathrm{n}^{\circ} 28$ : 58) ou les affections de la circulation veineuse (BV, 2003, $\left.\mathrm{n}^{\circ} 28: 45 ; \mathrm{AV}, 2005, \mathrm{n}^{\circ} 133: 32\right)$ qui peuvent atteindre les deux sexes mais qui touchent plus les femmes (Haesler et Hayoz, 2000).

Le recours à la célébrité, technique de marketing et stratégie publicitaire, est un autre procédé qui marque au féminin le discours du bien-être. Les lectrices, consommatrices potentielles, peuvent s'identifier à des reines du septième art, mais aussi à des femmes légendaires de l'Antiquité : «On peut [...] flotter dans la piscine «[m]er $[\mathrm{M}]$ orte» ou, plus glamour, jouer à Cléopâtre dans un bain de lait!» (AV, 2005, nº 133 :18).

Malgré ces procédés discursifs, ces magazines véhiculent majoritairement des discours non marqués, mais on a l'impression de pénétrer dans un univers féminin, de ressentir les effets d'un woman touch qui flotte plus spécialement dans les pages destinées à promouvoir le tourisme de bien-être. Quels sont alors les moyens par lesquels ils interpellent un public féminin, quelles sont donc les composantes de ce sceau féminin?

\section{Discours marqués : les textes et les images}

Tout d'abord, nous assistons à une mise en spectacle, véhiculée de façon solidaire par les discours journalistique et publicitaire, par le texte et les images, qui rend visibles les soins et les sensations éprouvées lors des séances dans les espaces bien-être. Le but final à obtenir est un bonheur extrême, un nirvana dérivé de l'équilibre, de l'harmonie physique et psychologique qui nous défend des agressions de la vie quotidienne. Ce parcours narratif vers une heureuse quiétude privilégié dans notre corpus ( $66 \%$ des discours rédactionnels et $20 \%$ des publicités payantes l'incorporent) est substitué par l'obtention de bénéfices bien plus concrets dans le discours promotionnel destiné aux hommes (Héraud, 2006).

L'expérience des cures est généralement vécue en individuel dans notre corpus (quand deux ou trois figures habitent un espace, elles ne sont jamais ensemble) et en couple de façon absolument exceptionnelle. Il n'en est pas de même quand il s'agit du séjour étant donné que les références aux familles et aux couples sont présentes dans les textes.

Le parcours individuel vers le bonheur absolu exige un espace et un temps pour soi. C'est justement la mise en spectacle du privé féminin qui est utilisée dans les magazines, une conception du privé revisitée. Domaine des femmes par excellence dans les systèmes de valeurs traditionnels, il dépasse le foyer pour s'installer dans le spa, le hammam, la cabine des soins, le «Jacuzzi»... Si le discours linguistique est en grande mesure neutre, le discours iconique est la plupart du temps marqué, puisque les photos avec figure représentent invariablement des femmes. Les signes iconiques et verbaux s'allient alors pour exhiber le spectacle intime de la femme consommatrice, pour montrer cette "parenthèse exquise» consacrée aux soins. Chaque photo fige à jamais un instant, où le temps devient non-temps, de ce parcours intime qui la conduira au bénéfice qui n'est plus sensoriel mais symbolique. C'est un parcours où la femme consommatrice, toujours la figure principale, est seule ou accompagnée du donneur de soins dont on ne voit souvent que les mains (voir illustration 1).

La mise en scène du spectacle de la consommation exige un décor dont la description minutieuse occupe une bonne partie de l'espace iconique et d'écriture. L'intimité indispensable à la réalisation individuelle de ce voyage intérieur exige la rupture physique et symbolique, la déconnexion du monde extérieur pour pénétrer dans un espace clos et silencieux, replié sur soi, une île de bonheur. Spécialement dans le cas des hôtels et des paquebots, la métaphore de l'île opère 
à plusieurs niveaux dans une structure en cercles concentriques qui se compose d'une macroscène qui forme un espace frontalier, d'une scène intermédiaire et finalement d'une microscène représentée par l'espace des soins où se produit la déconnexion absolue, la relaxation parfaite. Les différents lieux de passage de cette structure insulaire deviennent les symboles d'une rupture double, spatiale et temporelle, qui se trouve à la base du dépaysement. Ce procédé, qui favoriserait la mise en situation de la cible face au produit, que ce soit le rêve du tourisme ou le dépaysement par la lecture, expliquerait la multiplication des passages descriptifs consacrés à la décoration. L'influence des magazines de décoration, à la cible prioritairement féminine, y est intense par les emprunts d'éléments textuels et iconiques. C'est également un pôle d'attraction thématique pour les femmes et une manière subtile de marquer du genre féminin le discours neutre.

Arrêtons-nous sur les éléments qui constituent l'atmosphère dans l'espace des soins. Il faut signaler d'abord que, à l'instar de la description des différents traitements, tous les sens sont convoqués pour inspirer une ambiance douce, propice à la détente, à l'oubli total, à l'évasion : «La déco arabo-mauresque de bon aloi (mélange heureux de tadlack, de peinture à la chaux aux chaudes tonalités et de marbre). Les détails, la balnéo, la musique, les senteurs, les produits de soins, les bougies, les tisanes... " (AV, 2005, $\mathrm{n}^{\circ} 133$ : 32). Les décorations venues d'ailleurs, qui reprennent les traditions musulmanes ou zen, d'autres où le syncrétisme culturel est de mise, incitent spécialement au dépaysement. Comme dans les magazines de décoration, la palette de couleurs est en général très large et nuancée.

Un autre domaine d'interdiscursivité est à signaler car, très souvent, le discours consacré aux soins de bien-être et celui qui correspond aux produits de beauté pour femmes partagent, du moins partiellement, un jargon assez hermétique sauf pour les initiées. Les discours promotionnels montrent les mêmes tendances présentes depuis un certain temps dans les magazines voués aux femmes : le caractère scientifique de la dénomination des produits, la mise en valeur de l'utilisation de produits naturels ou les soins exotiques, traditionnels, dont on souligne l'authenticité, comme ceux qui viennent du nord de l'Afrique, de la Polynésie ou de l'Orient. C'est cette dernière tendance qui a le plus de visibilité dans notre corpus d'étude.

Notre analyse des discours marqués et non marqués a montré que l'absence de toute surdétermination du genre qu'un discours verbal neutre semblait véhiculer n'était qu'une stratégie de surface destinée probablement à ne pas exclure les lecteurs du sexe masculin déjà initiés aux services bien-être.

\section{Exotisme}

Le mythe de l'Ailleurs, construit sur des promesses et des non-dits, est caractérisé dans le discours promotionnel par l'omission, voire la négation de l'Autre (Cortés, Raskin et Turci, 2004; Cortés et Turci, 2007). On le retrouve parfois mais sa filiation est brève, faite d'un nom, d'une ethnie, d'une allusion à l'art thérapeutique dans lequel il, plutôt elle, excelle : des masseuses thaillandaises, un maître chinois du «feng-shui». Ces quelques sujets exotiques deviennent quand même les garants de l'étrange mélange de dépaysement et de déroute sensorielle induite qui parcourt aussi bien les textes que les images. Le sentiment d'exotisme, qui a toujours attiré les citadins cultivés, est imprégné ici des essences et des rites thérapeutiques millénaires venus d'Orient ou du Maghreb. Dans les textes, il est aussi garanti par la notion de tradition, sous forme de nom ou d'adjectif et par les dénominations étrangères des lieux, des techniques et des services. L'insistance sur ces formules contient un implicite qui, tout en dénonçant les pratiques intrusives, véhicule l'inquiétude et renforce l'attachement aux produits fiables, sécurisants, et de ce fait la tendance à idolâtrer les représentations de l'altérité et de son monde (Bensa, $2006: 341$ ).

Ailleurs revisité et domestiqué dont les actes sont décontextualisés et les acteurs dépersonnifiés (Bensa, 2006 : 15), "exotisme made in France», soins métis, l'esprit colonial et l'idéologie de la mondialisation s'entrelacent dans les représentations textuelles pour créer des espaces de quiétude et de ressourcement. Esthétique du Divers pour Ségalen (2004), l'exotisme, une fois les barrières de l'incompréhension apparemment effondrées, est conçu dans le discours promotionnel comme une pratique de la diversité dans un espace fermé, isolé du contexte et de l'Ailleurs et de l'Ici, où les décors, les produits et les soins servent à refermer l'étreinte qui préserve le bonheur des consommatrices.

\section{Sacralisation de l'espace bien-être}

Le discours du tourisme de bien-être, tout en conduisant son cœur de cible vers un microcosme allogène, vise à introduire une promesse de renouvellement en bâtissant un haut lieu de l'équilibre naturel, telle la fontaine de Jouvence. L'argument n'est pas neuf, puisque les vertus mythiques de l'eau étaient déjà abondamment mises en avant au XIX ${ }^{\mathrm{e}}$ siècle (Despine, 2003). Cependant, il ne s'agit plus de trouver la guérison, mais de ressaisir l'essence même de l'être à travers différents passages d'ordre tant psychique que physique. La première voie offerte est celle de la purification qui reprend par là le sens mystique de "rite destiné à mettre en état d'entrer en relation avec le divin» (Cnrs/Université Nancy 2/Atilf, 2002) : on «draine» les toxines ( $\left.\mathrm{BV}, 2003, \mathrm{n}^{\circ} 35: 55\right)$, on «évacue» le stress chronique (BV, 2003, $\mathrm{n}^{\circ} 28$ : 97). Les traitements conçus pour assurer une approche holistique qui parviennent à l'assainissement du corps et de l'esprit ne cessent de se renouveler afin de perpétuer la sensation que l'on bénéficie d'un privilège d'initié. Le second passage qui s'offre au public, conséquence logique du premier, est celui de la renaissance. Quête de perfection millénaire revivifiée sous l'égide des alchimistes et des chamans du passé ou sous la guidance moderne des philosophies orientales, cet itinéraire vise le rééquilibre, voire le renouveau des fonctions corporelles et mentales. L'imagerie mise en action est puissante. Toute une panoplie d'actions y est offerte pour atteindre la jeunesse éternelle. Sous forme adjective, «revigorant» (AV, 2003, $\mathrm{n}^{\circ} 122$ : 99), "régénérants» (AV, 2005, $\left.\mathrm{n}^{\circ} 136: 12\right)$, « restructurants » (BV, 2003, $\mathrm{n}^{\circ} 35$ : 64), elles conjuguent la visée prospective du participe présent avec la visée rétrospective induite par le préfixe «re-», ou bien elles expriment l'accomplissement libérateur du corps, toujours lui, dans la lutte contre le temps 


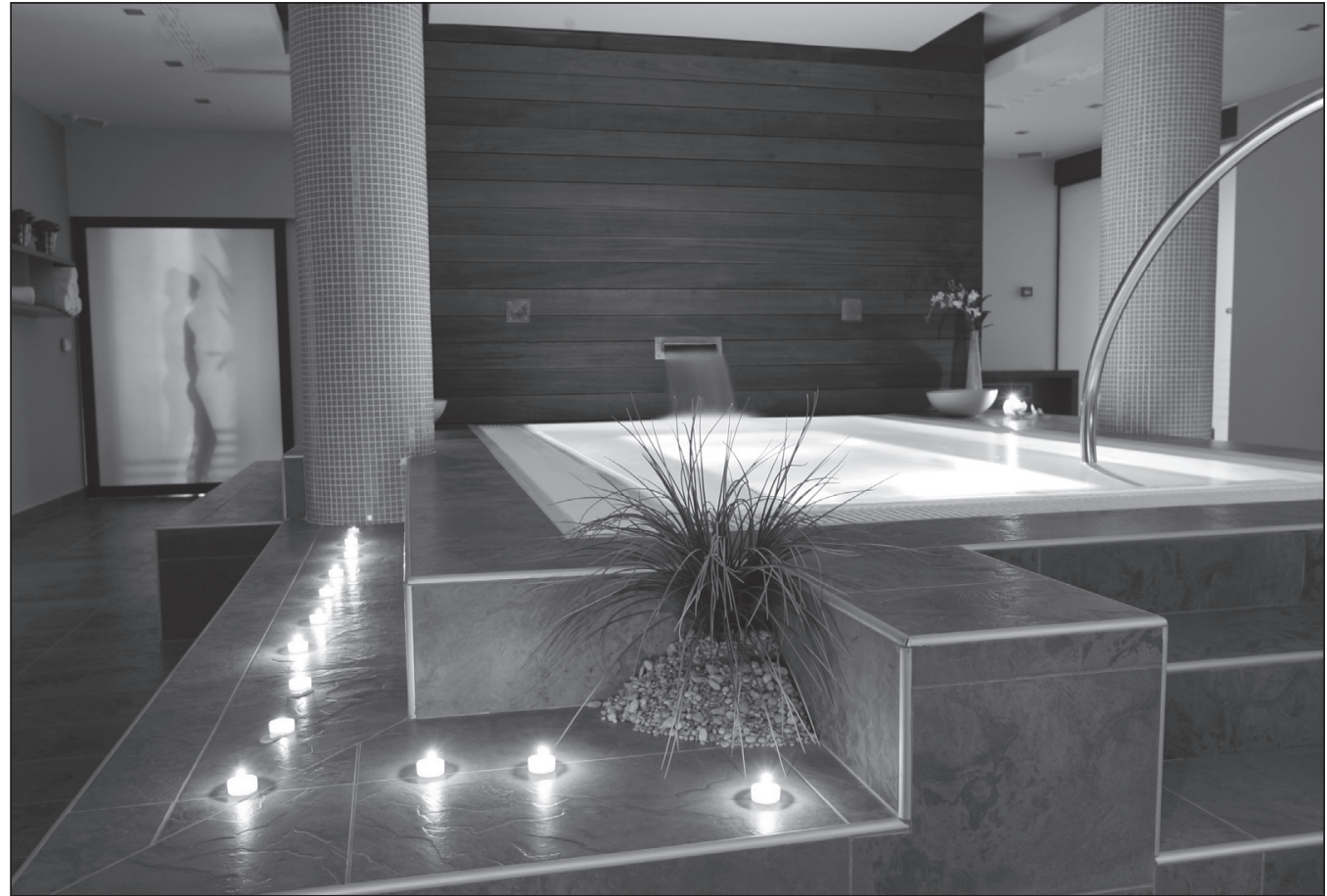

ILLUSTRATION 2 : L'espace des soins. Île de bonheur (source : Royalty Free Stock Images, Jerko). ou le stress au moyen du préfixe « dé-» et du participe passé, il est «détendu, déstressé, désintoxiqué» (AV, 2005, n 136 : 23). Une fois parachevé le sevrage personnalisé, le gommage, l'épilation ou le relooking d'urgence, l'accent est mis sur une peau «au grain affiné » (BV, 2003, $\left.\mathrm{n}^{\circ} 28: 44\right)$, sur des «jambes et un cœur léger $\left[\right.$ sic] » $\left(\mathrm{BV}, 2003, \mathrm{n}^{\circ} 28: 44\right)$, sur la disparition des symptômes de fatigue, sur le balayage de toute sensation de perte de vitalité, sur le renouvellement cellulaire "activé» (BV, 2003, $\left.\mathrm{n}^{\circ} 28: 52\right)$. Le but de ces paliers successifs n'est autre que le remodelage de la conscience identitaire. Ajustée à certains impératifs ataviques, dépendante d'arguments promotionnels omniprésents magnifiés à travers les médias, cette perception de soi est d'abord remise en cause pour être ensuite aiguillée vers des traitements hauts en couleur, voire thaumaturges. Évidemment, ce que nous désirons tous, hommes et femmes, se résume, dans le cadre du tourisme de bien-être, à la formule "pas d'esprit de cure mais des moments de bonheur» (BV, 2003, n 34 : 41). Le bonheur, intégré comme une visée globale tel qu'il est cité dans la Déclaration des droits de l'homme de 1789, souvent conçu comme un droit sous l'influence de la pensée américaine, est mis à notre portée le temps d'un séjour en spa. "Quelque chose comme une cathédrale dédiée au plaisir de l'eau»(AV, 1999-2000 : 86) (voir illustration 2), l'espace spa se voit de ce fait statufié, et les rites qui y ont lieu revêtus d'un caractère quasi sacré. Le spa devient sphère de culte "au-dessus des vicissitudes du monde» (AV, 1999-2000 : 146), où on officie avec une progression cérémonielle. C'est un besoin de sacralisation des sociétés modernes qui va de pair avec l'actualisation du credo identitaire, dont l'émergence croissante ne se limite plus au domaine des "cosmisations sacrées " (Hervieu-Léger et al., 1991 : 286-288), mais qui étend de plus en plus le versant symbolique du domaine touristique (David, 2007 : 143-154), étant donné que le tourisme est un moyen de se refaçonner, de faire naître un potentiel soi-même (Hasseler, 2008 : 19-26).

\section{Emprise de la tradition}

Les différentes études consacrées à l'iconographie des brochures touristiques réalisées dans les années 1990 par des chercheurs américains ont fait naître le concept de "gendered gaze» (Pritchard et Morgan, 2000 : 884-905). La vision sexuée que reproduit la promotion du tourisme dès ses débuts façonne un espace où s'opposent le masculin et le féminin dans la perception des destinations et des sites. Pritchard et Morgan (2000) soulignent les constitutifs patriarcaux et hétérosexuels de cette perspective traditionnelle selon laquelle les représentations de l'homme sont associées à l'action, à la propriété et au pouvoir, tandis que celles de la femme suggèrent passivité, disponibilité et dépendance. Il suffit de voir comment brochures et guides réintroduisent à l'envi l'optique néocoloniale qui fait des femmes natives aux traits racés - la japonaise en kimono, la négresse aux seins nus, la tahitienne en paréo - un symbole d'un pays. En un mot, la femme de l'Ailleurs, ainsi mise en disponibilité, devient l'accroche par antonomase du voyageur mâle et se pose comme l'antithèse hypersexuée de la cliente occidentale.

Au contraire, l'apport iconique de notre corpus met en avant de façon quasi exclusive un type de femme consommatrice intégrant les caractéristiques de peau claire, de jeunesse, de minceur, de beauté et de perfection corporelles. Idéal féminin contemporain véhiculé par la promotion, par le cinéma ou encore par la BD, il exclut de l'imagerie du tourisme de bien-être toute évocation de déviance du canon de beauté occidental et y nie la visibilité aux minorités 
ethniques en tant que consommatrices. Cette surexposition iconique réductrice remplit néanmoins un certain nombre de fonctions inhérentes au discours. L'image féminine apporte d'abord une représentation idéalisée de la cliente type blanche, mince et jeune, introduisant un élément de prestige et d'exclusivité qui imprègne l'espace des soins. C'est également une invitation à se confondre avec cet absolu visuel auquel la lectrice peut difficilement ne pas s'identifier.

Cela étant dit, il en va de manière distincte pour l'apport textuel. L'image de la femme devient composite au gré des associations systématiques qu'elle fait naître, des dualités symboliques qui la parcourent et des rôles socioculturels qui lui échoient.

La condition subalterne et dépendante de la femme traditionnelle semble revivre lorsqu'il lui est donné d'adopter une des positions rituelles, allongée, mi-nue et abandonnée aux mains expertes que dictent les traitements de bien-être.

La femme qui est ici mise en spectacle est vue à demiassise, de dos, en attente. Cette composition pourrait fort bien être confondue avec la perspective traditionnelle de $\mathrm{La}$ grande odalisque conçue par Ingres. Cela d'autant plus que les traitements que proposent les divers prestataires invitent à s'étendre et à «se laisser faire»: «Avec Aline, spécialisée en médecine traditionnelle chinoise et en acupression (digitopuncture), le contact est d'emblée agréable. Je me sens rapidement en confiance, prête à m'abandonner pour une première séance de réflexologie plantaire.» $\left(\mathrm{BV}, 2003, \mathrm{n}^{\circ} 28\right.$ : 46). Or, si la femme n'est plus seulement lectrice passive et image emblématique, elle le doit à sa liberté de choisir et à l'invitation qui lui est faite à travers le discours de «bien choisir». La passive sensualité est dès lors une option délibérément adoptée : «on aime s'y faire masser, dorloter» (BV, $\left.2003, \mathrm{n}^{\circ} 35: 71\right)$.

\section{Femme nouvelle}

La femme moderne, professionnelle, est fréquemment évoquée : «Zen millénaire pour stress contemporain. Ce soin [...] a pour but de décoincer dos, épaules et nuque des forçats de l'ordinateur!»(BV, 2003, no 35 : 97). La femme de carrière qu'elle est devenue peut s'approprier une partie de l'univers masculin, ainsi que quelques-unes de ses prérogatives. Certains éléments discursifs du bien-être reflètent clairement que des égards de préséance sont maintenant accordés à la femme effaçant le moindre vestige de doute quant à sa faculté de décider de son temps. On souligne à son intention la qualité des soins en apportant une description détaillée et valorisante des ingrédients. Le mieux-être est mis à sa portée, même quand il est nécessaire de minimiser le coût final, «[1] es moins : les prix élevés, mais que ne ferait-on pas pour être traité comme une VIP!» (BV, 2003, n 35 : 98).

La femme est poussée à l'action dans un rôle géminé qui superpose et entrelace les éléments masculins d'action et de pouvoir, et les éléments féminins de soumission, volontairement adoptée : «optez franchement pour une cure de remise en forme au centre de thalassothérapie» (AV, $2000: 78)$. La vision de la femme n'est donc plus ni conventionnelle ni linéaire : elle se complexifie et s'enrichit d'autant plus qu'elle devient porteuse d'une symbolique et d'une mythologie plurielles. D'un côté, se trouvent des décors-valeur qui, par un procédé métaphorique, transfèrent la qualité de leurs composantes à la prestation (Mendibil, 1989). D'un autre côté, c'est l'appel d'une riche mythologie orientalisante, parfois moderne lorsqu'on invoque Hollywood et ses fastes, «[p] our un traitement de reine qu'auraient aimé Greta Garbo ou Vivien Leigh» (AV, 2001, $\left.\mathrm{n}^{\circ} 109: 54\right)$.

Dans la partie textuelle qui se fait légende de l'icône, la femme objet devient conceptrice de l'image qu'elle veut projeter pour hommes et femmes. Ce nouveau pouvoir, acquis à l'extérieur du cercle clos de la famille, lui décerne un droit aux privilèges attenants, traditionnellement attribué aux hommes. Ces éléments satellites du pouvoir se matérialisent dans cet univers théâtralisé par l'élitisme du cadre, de l'équipement ou des traitements «spa haut de gamme au-dessus du lagon» (BV, 2002, $\left.\mathrm{n}^{\circ} 25: 53\right)$, ainsi que par la compétence du personnel traitant dont le seul prénom est synonyme de prise en charge personnalisée.

La mise en scène du luxe, décrit au moyen d'adjectifs appréciatifs, évoqué par des néologismes ou des mots étrangers, et visualisé dans des espaces à la grandeur et à la beauté calmes et enveloppantes, s'adresse à ces femmes non-dépendantes qui assument souvent un rôle prescriptif. Une nouvelle caste devenue miroir pour celles qui ne sont que la cible principale du discours promotionnel et qui peuvent se permettre un chic aller-retour en Hongrie le temps d'un bain. La nouvelle femme, forte et affranchie en société, apprécie de se laisser aller, de se laisser prendre en charge, de "se faire chouchouter» (BV, 2003, n $28: 48)$ en privé. Ces retraites où tous les sens sont convoqués, où elle prend conscience de son corps, lui redonnent de la vigueur et la réconcilient avec la vie, avec son être profond établi sur l'identité sociale (Bourdieu, 1998 : 92), contribuant en même temps à la conformation d'un habitus prestigieux. La sensualité des massages à plusieurs mains, souvent prises sur le vif dans les images, la volupté des enduits, des bains de boue, de vapeur ou de fleurs, des douches et même des séances de gymnastique aquatique, «le tout sur fond de musique douce» (BV, $2003, n^{\circ} 35$ : 98), l'amène à s'abandonner, un mot qui règne dans l'expression de ces transitivités, à la fois procès et état, où le sujet et l'objet ne font qu'un. Cependant, on peut aussi s'oublier totalement, jouir de nouvelles formes de ravissement, «se laisser envahir par une douce torpeur» (BV, 2003, $\mathrm{n}^{\circ} 28: 47$ ) dans une enveloppe d'algues, «s'engouffrer» (AV, 1999-2000 : 86) dans les bains, se perdre dans l'insondable, dans l'infini.

Malgré le caractère haut de gamme des soins de bien-être, ce modèle vise bien toutes les femmes, unies par un malêtre commun résultant de leur rôle pluriel dans la société : jambes lourdes, stress, grossesse, les autres dérèglements du corps ou de l'esprit que ce soient les varices, le surpoids ou la déprime étant tabous. Elle mérite le bien-être ou plutôt le mieux-être, elle est déculpabilisée au moyen d'arguments qui reviennent régulièrement dans les textes : d'abord parce qu'elle le vaut, puis parce qu'elle en a besoin pour assumer son rôle de femme-mère, femme-professionnelle, femme-épouse, etc. d'une façon efficace, mais aussi par l'intégration de la femme dans une culture de la Femme, 
autochtone, planétaire, atemporelle. Dans un article destiné aux agents de voyage, Françoise Mommens (2005) donne ce conseil : «Les femmes aiment s'octroyer de petits luxes et des moments d'évasion. Vendez-leur du plaisir. Offrez-leur des instants privilégiés — pris au détriment du « devoir» qui leur serviront à se récompenser, mais déculpabilisez-les de le faire.» Tout l'amène donc au plaisir, au bonheur, elle y est même contrainte.

\section{Conclusion}

Le discours des magazines classés «tourisme et voyage » s'est avéré représenter un support d'interpellation directe de la femme. Pourtant, les indices verbaux féminins, rares dans nos textes, ne pouvaient pas expliquer notre hypothèse. Une approche sémiotique qui tienne compte de la composition des discours et de l'hybridité des signes qui articulent le message pouvait nous donner les outils d'analyse afin de faire émerger les procédés particuliers de marquage de genre qui sous-tendent le texte et en orientent l'interprétation. Cette démarche, centrée sur le discours du tourisme de bien-être, révèle pour la première fois les stratégies, les ressorts sousjacents de persuasion qui font de la femme le cour de cible de ces magazines.

Les réseaux de sens qui se tissent par l'interaction entre les images et les textes, et ceux émanant des résonances intertextuelles ancrent le discours neutre dans un univers essentiellement féminin. Le maillage argumentatif est orienté dans le sens de la promesse d'un bonheur absolu qui comblerait les désirs de la femme d'aujourd'hui. Cette promesse est garantie par des services et des produits exclusifs et véhiculée par des thèmes récurrents. L'exotisme devient évocation domestiquée de l'Autre à la mesure du regard occidental dans un espace concentrique, isolé du monde, permettant une évasion et un bonheur contrôlés. Ceci exige une mise en spectacle du privé qui sacralise l'espace bien-être où les soins sont ritualisés. La femme consommatrice, quant à elle, est présentée de manière paradoxale : réceptrice passive des soins dans les images, elle se voit investir des rôles traditionnellement attribués à la femme tout en régnant sur cette sphère sécurisante qu'elle a librement choisie.

\section{Références}

BENSA, Alban (2006) La fin de l'exotisme, Toulouse : Anacharsis. 372 p. BOURDIEU, Pierre (1990) Le sens pratique, Paris : Minuit. 474 p. BOURDIEU, Pierre (1998) La domination masculine, Paris : Le Seuil. 174 p. BUGNOT, Marie-Ange (2009) Le discours touristique ou la réactivation du locus amoenus, Grenade : Comares. 165 p.

CALIANDRO, Dominique (2004) «'Beauté’ : des rédactrices de magazines féminins encore pires que les publicitaires", Acrimed, $<\mathrm{http}: / / \mathrm{www}$. acrimed.org/article1449.html>, consulté le 17 mars 2007.

CHEN, Joseph S.; Nina PREBENSEN et T. C. HUAN (2008) «Determining the Motivation of Wellness Travelers», Anatolia: An International Journal of Tourism and Hospitality Research, vol. 19, n 1, p. 103-115.

CHEVRIER, François G. (2006) « Les copains ensuite... Que devient l'univers masculin du voyage?», Réseau de veille en tourisme, Chaire de tourisme Transat de l'École des sciences de la gestion de l'Université du Québec à Montréal, <veilletourisme.ca/docs/globeveilleur_090806.pdf>, consultéle 28 janvier 2010.
CNRS/UNIVERSITÉ NANCY 2/ATILF (2002) Le Trésor de la Langue Française informatisé, version $4,<$ http://atilf.atilf.fr/tlfv3.htm $>$, consulté le 12 décembre 2009.

CORTÉS, Carmen; Lydia RASKIN et Isabel TURCI (2004) «La negación de la alteridad en las revistas de viajes", DANS L'Autre et soi-même. La identidad y la alteridad en el ámbito Francés y Francófono, Me Pilar SUÁREZ, Margarita ALFARO, André BÉNIT, Patricia MARTÍNEZ, Carmen MATA et Didier TEJEDOR (éd.), p. 357-367. Madrid : UAM.

CORTÉS, Carmen et Isabel TURCI (2007) «La construcción del sujeto en las revistas de viajes", DANS Interculturalidad y Lenguaje II. Identidad cultural y pluralidad lingüística, Juan de Dios LUQUE DURÁN et Antonio PAMIES BELTRÁN (éd.), p. 65-73. Granada : Granada Lingvistica.

CORTESE, Anthony (2008) Provocateur: images of women and minorities in advertising, Plymouth : Rowman \& Littlefield.179 p.

COURTÉS, Joseph (s.d.) «La sémiotique de l'École de Paris», <http:// w3.gril.univ-tlse2.fr/SEM.html>, consulté le 12 octobre 2010.

DAVID, Béatrice (2007) «Tourisme et politique : la sacralisation touristique de la nation en Chine», Hérodote, vol. 2, n 125, p. 143-156.

DESPINE, Philippe (2003) «Thermalisme et civilisation : entre rationalité et imaginaire», La Presse thermale et climatique, $n^{\circ} 140$, p. 53-59.

DUTHEIL, Frédéric (2003) «Le sport à la cure: le corps médical face à la diffusion des pratiques sportives dans la station thermale de Vichy. 1875$1914 »$, Staps, n 61, p. 39-52.

ECO, Umberto (1991) Tratado de semiótica general, Barcelona : Blumen. $461 \mathrm{p}$.

EUROMONITOR INTERNATIONAL (2009a) «Health \& Wellness Tourism - Switzerland», $<$ http://www.euromonitor.com/Travel_and_Tourism_ in_Switzerland >, consulté le 13 janvier 2010.

EUROMONITOR INTERNATIONAL (2009b) «Health \& Wellness Tourism — France», $<$ http://www.euromonitor.com/Travel_and_Tourism_in_ France $>$, consulté le 13 janvier 2010.

EVERAERT-DESMEDT, Nicole (1990) Le processsus interprétatif, Liège : Mardaga. 153 p.

EVERAERT-DESMEDT, Nicole (2000) Sémiotique du récit, Bruxelles : De Boeck-Université. 323 p.

EVERAERT-DESMEDT, Nicole (2006) «La sémiotique de Peirce», DANS Signo, sous la direction de Louis HÉBERT, $<$ http://www.signosemio. com/>, consulté le 9 octobre 2009.

FONTANARI, Martin et Alexandra KERN (2003) «The "Comparative Analysis of Spas" - An Instrument for the Re-Positioning of Spas in the Context of Competition in Spa - and Health Tourism ", Tourism Review, vol. $58, \mathrm{n}^{\circ} 3$, p. 20-28.

GARCÍA-ALTÉS, Ana (2005) «The Development of Health Tourism Services", Annals of Tourism Research, vol. 32, nº 1, p. 266-268.

GLOBAL SPA SUMMIT (2010) «Spas and the Global Wellness Market: Synergies and Opportunities, prepared by SRI International, May 2010», <gss_sri_spasandwellnessreport_rev_82010.pdf>, consulté le 10 octobre 2010.

GROS, Jean-François (1899) Vichy et ses environs. Guide-poche, $28^{e}$ année, été, Cusset : Impr. de J. Arloing et M. Bouchet, cité dans DUTHEIL, Frédéric (2003) «Le sport à la cure: le corps médical face à la diffusion des pratiques sportives dans la station thermale de Vichy. 1875-1914», Staps, $\mathrm{n}^{\circ}$ 61, p. 39-52.

HAESLER, Erik et Daniel HAYOZ (2000) «Heavy legs and hormone replacement therapy», Sang Thrombose Vaisseaux, vol. 12, n 7, p. 433-9, $<$ http://www.john-libbey-eurotext.fr/en/revues/medecine/bdc/edocs/00/03/D3/0E/article.phtml>, consulté le 12 octobre 2010. 
HASSELER, Terry A. (2008) «The Promise of Tourism: Colonial Imagery in Advertising», Radical Teacher, $\mathrm{n}^{\circ} 82$, p. 19-26.

HÉRAUD, Béatrice (2006) «Le marketing a t’il un sexe?», Marketing Magazine, $\mathrm{n}^{\circ}$ 108, <http://www.e-marketing.fr/Marketing-Magazine/ Article/Le-marketing-a-t-il-un-sexe--17775-1.htm>, consulté le 24 novembre 2007.

HERVIEU-LÉGER, Danièle; Claude RIVIERE et Albert PIETTE (éd.) (1991) «Nouvelles idoles. Nouveaux Cultes. Dérives de la sacralité », Archives des sciences sociales des religions, vol. 74, $\mathrm{n}^{\circ} 1$, p. 286-288.

IPSOS (2009) «Le marché du bien-être», <http://www.ipsos.fr/>, consulté le 31 juillet 2009.

L'ASSEMBLEE DES FEMMES. LANGUEDOC-ROUSSILLON (2003) "La force des femmes au service du tourisme», <http://www.assemblee-des-femmes.com/contacts/languedoc/tourisme.pdf $>$, consulté le 11 octobre 2010.

LE BOEUF, Claude (2000) «Réflexion sur le métissage de la sémiotique, du marketing et de la science de la communication à propos du statut des produits-signes », Tangences, $\mathrm{n}^{\circ}$ 64, p. 49-61.

LEFEBVRE, Anne (1999-2000) Art de voyager, Puteaux : YTC Publishing, $\mathrm{n}^{\circ} 97$.

LEFEBVRE, Anne (2000) Art de voyager, Puteaux : YTC Publishing, n 103.

LEFEBVRE, Anne (2001) Art de voyager, Puteaux : YTC Publishing, no 108 et 109 .

LEFEBVRE, Anne (2002) Art de voyager, Puteaux : YTC Publishing, nº 114 à 119.

LEFEBVRE, Anne (2003) Art de voyager, Puteaux : YTC Publishing, nº 120 à 123.

LEFEBVRE, Anne (2005) Art de voyager, Puteaux : YTC Publishing, nº 133 et 136 .

LEFEBVRE, Anne (2006) Art de voyager, Puteaux : YTC Publishing, nº 138.

MAHÉ, Patrick (2002) Bon voyage, Levallois-Perret : Hachette Filipacchi, $\mathrm{n}^{\circ} 25$ et 26 .

MAHÉ, Patrick (2003) Bon voyage, Levallois-Perret : Hachette Filipacchi, $\mathrm{n}^{\circ} 27$ à 36.

MAHÉ, Patrick (2004) Bon voyage, Levallois-Perret : Hachette Filipacchi, $\mathrm{n}^{\circ} 43$.

MAURIN, Véronique (2003) «Vrais catalogues, faux magazines : la presse féminine sort ses 'magalogues' », Acrimed, <http://www.acrimed.org/ article1390.html>, consulté le 10 février 2005.

MENDIBIL, Didier (1989) «Publicité et géographie : paysages, images et discours », Strates, $\mathrm{n}^{\circ}$ 4, <http://strates.revues.org/document4162.html>, consulté le 16 février 2010.

MERMET, Gérard (2010) Francoscopie. Tout sur les français, Paris : Larousse. 539 p.
MOMMENS, Françoise (2005) «Savez-vous parler aux femmes?», Réseau de veille en tourisme, Chaire de Tourisme Transat de l'École des sciences de la gestion de l'Université du Québec à Montréal, <http://veilletourisme.ca/2005/05/03/savez-vous-parler-aux-femmes $>$, consulté le 27 janvier 2010.

PERRET, Jean-Baptiste (2003) «L'approche française du genre en publicité», Réseaux, vol. 4, n 120, p. 147-173, <www.cairn.info/revue-reseaux2003-4-page-147.htm>, consulté le 20 juin 2008.

PRITCHARD, Annette et Nigel J. MORGAN (2000) «Privileging the Male Gaze - Gendered Tourism Landscapes », Annals of Tourism Research, vol. $27, \mathrm{n}^{\circ} 4$, p. $884-905$.

PROULX, Luce (2005) «Tourisme, santé et bien-être», Téoros, vol. 24, n³, automne, p. 5-11.

REISER Michèle et Brigitte GRESY (2008) L'image des femmes dans les médias. Paris : La Documentation française. 95 p.

RIBAS, Montserrat et Júlia TODOLÍ (2008) «La metáfora de la mujer objeto y su reiteración en la publicidad», Discurso y Sociedad, vol. 2, n 1 , p. $153-169$.

ROSS, Kim (2001) «Health Tourism: An Overview», HSMAI Marketing Review, <http://www.hospitalitynet.org/news/4010521.search?query=\% 22 health+tourism\%22>, consulté le 13 avril 2006.

ROUGÈS, Jean-François (2009) «La femme est l'avenir du marketing», Curiosités stratégiques, <http://curiosites-strat.blogspot.com/2009/09/ la-femme-est-lavenir-du-marketing.html>, consulté le 11 octobre 2010.

SEGALEN, Victor (2004) Une esthétique du divers, Saint Clément de Rivière : Fata Morgana. 104 p.

SMITH, Melanie et Lászlo PUCZKÓ (2009) Health and Wellness Tourism, Oxford : Butterworth-Heinemann. 400 p.

THERMALIES (2010) «Spa, thalassothérapie...le bien être pour $49 \%$ des Français », <http://www.thermalies.com/IMG/pdf/CPno6.pdf >, consulté le 18 février 2010.

TISSIER-DESBORDES, Elisabeth et Isabelle ULRICH (2006) «Masculinféminin : les atouts de la segmentation par genre», Les Echos, n 19805 , p. 110, <http://archives.lesechos.fr/archives/2006/LesEchos/19805-508ECH.htm>, consulté le 20 novembre 2009.

WALLON, Armand (1981) La vie quotidienne dans les villes d'eaux (18501914), Paris : Hachette. 349 p.

WEARING, Betsy et Steven WEARING (1996) «Refocusing the Tourist Experience. The "Flaneur" and the "Choraster" ", Leasure Studies, n" 15, p. 229-244, cité dans PRITCHARD, Annette et Nigel J. MORGAN (2000) «Privileging the Male Gaze — Gendered Tourism Landscapes », Annals of Tourism Research, vol. 27, $\mathrm{n}^{\circ}$ 4, p. 884-905.

WYKES, Maggie et Barrie GUNTER (2005) The Media and Body Image. New Castle : SAGE, 252 p. 